\title{
Using Biased Support Vector Machine to Improve Retrieval Result in Image Retrieval with Self-organizing Map
}

\author{
Chi-Hang Chan and Irwin King \\ Department of Computer Science and Engineering \\ The Chinese University of Hong Kong \\ Shatin, N.T., Hong Kong \\ \{chchan, king\}@cse.cuhk. edu.hk
}

\begin{abstract}
The relevance feedback approach is a powerful technique in content-based image retrieval (CBIR) tasks. In past years, many intraquery learning techniques have been proposed to solve the relevance feedback problem. Among these techniques, Support Vector Machines (SVM) have shown promising results in the area. More specifically, in relevance feedback applications the SVMs are typically been used as binary classifiers with the balanced input data assumption. In other words, they do not consider the imbalanced dataset problem in relevance feedback, i.e., the non-relevant examples outnumbered the relevant examples. In this paper, we propose to apply our Biased Support Vector Machine (BSVM) to address this problem. Moreover, we apply our Self-Organizing Mapbased inter-query technique to reorganize the feature vector space, in order to incorporate the information provided by past queries and improve the retrieval performance for future queries. The proposed combined scheme is evaluated against real world data with promising results demonstrating the effectiveness of our proposed approach.
\end{abstract}

\section{Introduction}

The goal of relevance feedback is to learn user's preference from their interaction, and it is a powerful technique to improve the retrieval result in CBIR. Under this framework, a set of images is presented to the user according to the query. The user marks those images as either relevant or non-relevant and then feeds back this into the system. Based on these feedback information, the system presents another set of images to the user. The system learns user's preference through this iterative process, and improves the retrieval performance.

Most of the current relevance feedback systems are based on the intra-query learning approach [1-3]. In this approach, the system refines the query and improves the retrieval result by using feedback information that the user provided. Recently, researchers introduced the regular SVM [1] and one-class SVM [2] into the relevance feedback process, and it shows that SVM-based techniques are more promising and effective techniques than other intra-query approaches. The regular SVM [4] technique treats the relevance feedback problem as a strict 
binary classification problem. However, this technique does not consider the imbalanced dataset problem, in which the number of non-relevant images are significantly larger than the relevant images. This imbalanced dataset problem will lead the positive data (relevant images) be overwhelmed by the negative data (non-relevant images). The one-class SVM [5] uses only the relevant images in the learning process, and treats the problem as a density estimation problem. The one-class SVM technique seems to avoid the imbalanced dataset problem. However, it cannot work well without the help of negative information.

Recently, researchers propose the use of inter-query information to further improve retrieval result $[6,7]$. In the inter-query approach, feedback information from past queries are accumulated to train the system to determine what images are of the same semantic meaning. These approaches show that the retrieval performance can be benefited from the inter-query learning.

In this paper, we propose a relevance feedback technique to incorporate both inter-query and intra-query information for modifying the feature vector space and estimating the users' target. Self-Organizing Map (SOM) [8] is used to cluster and index the images in the database. We apply our SOM-based inter-query technique [6] to modify the feature vector space, in which the SOM of images is stored. This allows for transforming the images distributions and improving their organization in the modified vector space. Moreover, we propose to apply our BSVM [9] technique to capture the user's individual preferences in the relevance feedback process, and address the imbalanced dataset problem in relevance feedback process.

\section{Biased Support Vector Machine}

Our Biased Support Vector Machine (BSVM) is derived from 1-SVMs, and the objective of BSVM is to describe the data by employing a pair of sphere hyperplanes in which the inner one captures most of the relevant data while the outer one pushes out the non-relevant data. Moreover, the distance between these two sphere hyperplanes have to be maximized. The task can be formulated as an optimization problem and the mathematical formulation of our technique is given as follows.

The objective function for finding the optimal sphere hyperplane can be formulated below:

$$
\begin{array}{cl}
\min _{R \in \mathbb{R}, \xi \in \mathbb{R}, \rho \in \mathbb{R}} & b R^{2}-\rho+\frac{1}{n \nu} \sum_{i=1}^{n} \xi_{i}, \\
\text { s.t. } & y_{i}\left(\left\|\Phi\left(\mathbf{x}_{\mathbf{i}}\right)-\mathbf{c}\right\|^{2}-R^{2}\right) \leq-\rho+\xi_{i}, \\
& b \geq 0, \xi_{i} \geq 0, \rho \geq 0,0 \leq \nu \leq 1,
\end{array}
$$

where $\xi_{i}$ are the slack variables for margin error, $\Phi\left(\mathbf{x}_{\mathbf{i}}\right)$ is the mapping function, c and $R$ are the center and radius of the optimal hypersphere, $\rho$ is the width of the margin, $b$ is a parameter to control the bias, and $\nu \in[0,1]$ is a parameter to control the tradeoff between the number of support vectors and margin errors. 
The optimization task can be solved by introducing the Lagrange multipliers. The dual of the primal optimization can be shown to take the form

$$
\begin{gathered}
\max _{\alpha} \sum_{i} \alpha_{i} y_{i} k\left(\mathbf{x}_{\mathbf{i}}, \mathbf{x}_{\mathbf{i}}\right)-\frac{1}{b} \sum_{i, j} \alpha_{i} \alpha_{j} y_{i} y_{j} k\left(\mathbf{x}_{\mathbf{i}}, \mathbf{x}_{\mathbf{j}}\right) \\
\text { s.t. } \sum_{i} \alpha_{i} y_{i}=b, 0 \leq \alpha_{i} \leq \frac{1}{n \nu}, \sum_{i} \alpha_{i} \geq 1
\end{gathered}
$$

This dual problem can be solved with quadratic programming techniques.

The way we construct the BSVM is efficient for solving the imbalanced dataset problem in relevance feedback. Since the weight allocated to the positive support vectors in BSVM will be larger than the negative ones when setting a positive bias factor $b$. This can be useful for solving the imbalanced dataset problem. However, regular SVMs treat the two classes without any bias which is not effective enough to model the relevance feedback problem. Moreover, we fully utilize both positive and negative data in the training process.

\section{Proposed Algorithm}

\subsection{Preprocessing}

In the preprocessing procedure, the system performs feature extraction on the images $I$ in the database, and uses a SOM to represent the distribution of the data. We perform a low-level feature extraction on the set of images in the database, and each image is then represented by a feature vector $\mathbf{x}_{i} \in \mathbb{R}^{d}$ in a high dimensional vector space. We construct and train a SOM $M$ with feature vectors extracted from the images. After the SOM training, the model vectors in the neurons of $M$ are arranged to match the distribution of the feature space. The model vectors $\mathbf{m}_{i} \in M$ of neurons in the SOM are used to partition the feature vector space based on the minimum distance classifier, each image $I_{i}$ is classified into different groups represented by $\mathbf{m}_{i}$.

\subsection{Intra-query Learning}

In the intra-query learning process, the system presents a set of images $D_{t}$ to the user in each iteration $t$, and the user gives response $A_{t}$ by marking them as either relevant or non-relevant. The information provided in the $k$-th query at iteration $t$ is represented by $q_{t}^{k}=\left\{D_{1}, A_{1}, \ldots, D_{t}, A_{t}\right\}$, and the system uses it to refine the query. We define $D_{R}$ and $D_{N}$ as the set of relevant images and the set of non-relevant images marked by the user from first iteration to the current iteration respectively. The sets $D_{R}$ and $D_{N}$ are then represented by the corresponding model vector set $M_{R}$ and $M_{N}$. The BSVM in Section 2 is used to train a decision boundary to classify this two sets of data.

In order to retrieve images from the database, we need to construct a evaluation function to output the relevance value of the neurons, and it is defined by,

$$
g\left(\mathbf{m}_{i}\right)=R^{2}-\left\|\Phi\left(\mathbf{m}_{i}\right)-\mathbf{c}\right\|^{2}
$$


where the radius $R$ and center $\mathbf{c}$ can be solved by a set of support vectors, and it can be done by solving the Eq. (4). The neurons with higher scores will be more likely to be chosen as the targets. The relevance score between an image and its corresponding neutron is measured by their Euclidean distance. Thus, we can rank the images in the database by combining it with the function $g\left(\mathbf{m}_{i}\right)$.

\subsection{Inter-query Learning}

In most of the relevance feedback systems, only the intra-query feedback information is used to learn the user's preference. However, a small training data set is difficult to provide enough statistical information for achieving this goal and providing good retrieval result. In order to address this problem, we use the inter-query information to modify the feature vector space and cluster the neurons with similar images together, so that the neutrons are organized in a way that ease the process of intra-query learning.

In order to update the similarity measure based on the inter-query feedback information, we modify the model vectors $\mathbf{m}_{i}$ in the new SOM, such that neurons contain similar images as indicated in the feedback are moved closer to each others. Consider that there are $K$ past queries stored in the system, and interquery information provided to the system is represented by $\left\{q^{1}, \ldots, q^{K}\right\}$. Each past query $q^{k}$ is used to reorganize the vector space of the SOM, and improve the structure of data. Assume in the $k$-th query, the user marked a set of relevant images $D_{R}^{k}$ and a set of non-relevant images $D_{N}^{k}$ during the whole retrieval process, $M_{R}^{k}$ and $M_{N}^{k}$ are the corresponding sets of model vectors respectively. Let $\mathbf{c}^{\prime k}$ be the model vector with highest relevance score in Eq. (6), and it is most likely to be the user's target for that query. In our SOM-based inter-query learning, neurons represent relevant images are moved closer to the estimated user's target and those represent non-relevant images are moved away from the estimated user's target. For a long run, the vector space will be modified, in which neurons represent the same image concept are clustered together.

In a SOM, the nearby neurons in the topology are representing similar units, so that the learning process can be improved by moving also the neurons that near to the neurons in the sets $M_{R}^{k}$ and $M_{N}^{k}$. The idea of this process is similar to the SOM training process. The equations for modifying the model vectors are defined by,

$$
\begin{aligned}
& \forall \mathbf{m} \in N\left(M_{R}^{k}\right), \mathbf{m}=\mathbf{m}+h_{R i}^{k}\left(\mathbf{c}^{\prime k}-\mathbf{m}\right), \\
& \forall \mathbf{m} \in N\left(M_{N}^{k}\right), \mathbf{m}=\mathbf{m}+h_{N i}^{k}\left(\mathbf{m}-\mathbf{c}^{\prime k}\right),
\end{aligned}
$$

where $N(M)$ is the set of nearby neurons for $M$ in the SOM topology. $h_{R c i}^{k}$ and $h_{N c i}^{k}$ are the neighborhood functions; they are monotonic decreasing function with $k$ and the distance with the corresponding model vector in $M_{R}^{k}$ or $M_{R}^{k}$.

\section{Experiment}

Here, we present the setup of our experiments on various relevance feedback systems for CBIR. The relevance feedback systems involved in the experiment are 
MARS [3], 1-SVM [2], $\nu$-SVM [10] and our proposed method. For the purpose of objective measure of performance, we assume that the query judgement is defined on the image categories. The metric of evaluation is the average precision. The dataset used in the experiment is the real-world images chosen from the COREL image collection. The datasets is with 50 categories. Each category includes 100 images belonging to a same semantic class. We extract three different features to represent the images: color, shape and texture. We combine this three different features as a 36-dimensional feature space.

In the first experiment, we evaluate the retrieval performance of four intraquery methods. A category is first picked from the database randomly, and this category is assumed to be the user's query target. The system then improves retrieval results by relevance feedbacks. In each iteration of the relevance feedback process, five images are picked from the database and labelled as either relevant or non-relevant based on the ground truth of the database. The precision of each method is then recorded, and the whole process is repeated for 200 times to produce the average precision in each iteration for each method. Fig. 1 shows the evaluation result on the top-30 average precision.

In the second experiment, we evaluate the retrieval performance of our SOMbased inter-query learning technique by applying it to those intra-query learning techniques in the first experiment. In this experiment, the feature vectors of images in the database are used to train a SOM of dimension of $30 \times 30$. The 200 queries generated in the first experiment are used to further train the SOM with our proposed inter-query learning technique. Finally, we generated another 200 queries and recorded the precision of each method. We evaluate the performance of our inter-query learning by comparing the retrieval result with the first experiment. We list the top-30 average precision at various iteration in Table 1.

From the experiment, the BSVM outperforms the other three intra-query techniques. Typical approaches by SVMs without considering the bias in the retrieval tasks is not reasonable and good enough to solve the relevance feedback problem. We also see that regular one-class SVMs do not consider the negative information which cannot learn the feedback well. Moreover, all four intra-query techniques perform better when our SOM-based inter-query learning technique is

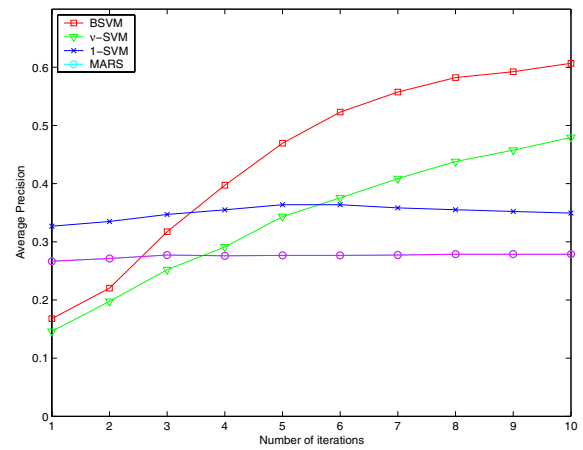

Table 1. Top 30 Average Precision with SOM

\begin{tabular}{|c|c|c|c|c|c|}
\hline Iteration & 1 & 3 & 5 & 7 & 9 \\
\hline MARS & 0.267 & 0.277 & 0.277 & 0.277 & 0.279 \\
\hline MARS & 0.285 & 0.296 & 0.298 & 0.298 & 0.301 \\
\hline 1-SVM & 0.327 & 0.347 & 0.363 & 0.358 & 0.352 \\
\hline $1-$ SVM & $\mathbf{0 . 3 6 5}$ & $\mathbf{0 . 3 7 6}$ & 0.379 & 0.366 & 0.371 \\
\hline$\nu$-SVM & 0.146 & 0.252 & 0.343 & 0.409 & 0.458 \\
\hline$\nu$-SVM & 0166 & 0.291 & 0.376 & 0.451 & 0.465 \\
\hline BSVM & 0.168 & 0.317 & 0.470 & 0.558 & 0.592 \\
\hline BSVM & 0.191 & 0.359 & $\mathbf{0 . 5 1 5}$ & $\mathbf{0 . 6 0 2}$ & $\mathbf{0 . 6 1 1}$ \\
\hline
\end{tabular}

Fig. 1. Top 30 Average Precision 
applied, and it performs the best when incorporate with our BSVM. It shows that the SOM-based inter-query learning can help the intra-query learning process and improve the retrieval result.

\section{Conclusion}

In this paper, we investigate SVMs techniques for solving the relevance feedback problems in CBIR. We address the imbalanced dataset problem in relevance feedback and propose a novel relevance feedback technique with Biased Support Vector Machine. The advantages of our proposed techniques are explained and demonstrated compared with traditional approaches. Moreover, we propose a SOM-based inter-query learning technique to reorganize the feature vector space, such that the information provided in past queries is utilized and the retrieval result is improved. We perform experiments on real-world image datasets. The experimental results demonstrate that our BSVM-based relevance feedback algorithm and SOM-based inter-query learning technique are effective and promising for improving the retrieval performance in CBIR.

\section{Acknowledgement}

This work is supported in part by the RGC Research Grant Direct Allocation \#2050259.

\section{References}

1. Tong, S., Chang, E.: Support vector machine active learning for image retrieval. In: Proceedings of the ninth ACM international conference on Multimedia, ACM Press (2001) 107-118

2. Chen, Y., Zhou, X., Huang, T.S.: One-class svm for learning in image retrieval. In: Proceedings of the IEEE international conference on Image Processing. (2001) $34-37$

3. Rui, Y., Huang, T.S., Ortega, M., Mehrota, S.: Relevance feedback: A power tool for interactive content-based image retrieval. IEEE Transactions on Circuits and Video Technology (1998) 644-655

4. Vapnik, V.N.: The Nature of Statistical Learning Theory. Springer (1995)

5. Tax, D.M.J., Duin, R.P.W.: Support vector domain description. Pattern Recignition Letters 20 (1999) 1191-1199

6. Chan, C.H., Sia, K.C., King, I.: Utilizing inter and intra-query relevance feedback for content-based image retrieval. In: Special Session of Joint 13th International Conference on Artificial Neural Network and 10th International Conference on Neural Information Processing. (2003)

7. He, X., King, O., Ma, W.Y., Li, M., Zhang, H.J.: Learning a semantic space from user's relevance feedback for image retrieval. IEEE Transactions on Circuits and Systems for Video Technology 13 (2003) 39-48

8. Oja, E., Kaski, S.: Kohonen Maps. Hardbound (1999)

9. Hoi, C.H., Chan, C.H., Huang, K., Lyu, M.R., King, I.: Biased support vector machine for relevance feedback in image retrieval. In: Proceedings of the International Joint Conference on Neural Networks (IJCNN'04) accepted. (2004)

10. Scholkopf, B., Smola, A., Williamson, R., Bartlett, P.L.: New support vector algorithms. Neural Computation 12 (2000) 1207-1245 